\title{
DIAMETRICALLY CONTRACTIVE MAPS AND FIXED POINTS
}

\author{
MARCO BARONTI, EMANUELE CASINI, AND PIER LUIGI PAPINI
}

Received 13 January 2006; Revised 3 May 2006; Accepted 3 May 2006

Contractive maps have nice properties concerning fixed points; a big amount of literature has been devoted to fixed points of nonexpansive maps. The class of shrinking (or strictly contractive) maps is slightly less popular: few specific results on them (not applicable to all nonexpansive maps) appear in the literature and some interesting problems remain open. As an attempt to fill this gap, a condition half way between shrinking and contractive maps has been studied recently. Here we continue the study of the latter notion, solving some open problems concerning these maps.

Copyright (c) 2006 Marco Baronti et al. This is an open access article distributed under the Creative Commons Attribution License, which permits unrestricted use, distribution, and reproduction in any medium, provided the original work is properly cited.

\section{Introduction}

Let $X$ be a Banach space and $M$ a nonempty convex closed bounded subset of $X$. In the theory of fixed points, two classes of maps $T: M \rightarrow M$ are well known and deeply studied: the class of contractive maps

$$
\forall x, y \text { in } M, \quad\|T x-T y\| \leq \alpha\|x-y\|, \quad \alpha \in(0,1)
$$

and the class of nonexpansive maps

$$
\forall x, y \text { in } M, \quad\|T x-T y\| \leq\|x-y\| .
$$

An intermediate class consists of the maps that satisfy the following condition:

$$
\|T x-T y\|<\|x-y\| \quad \forall x \neq y \text {, with } x, y \in M \text {. }
$$

In the literature, these maps appear under different names, see for example [5] and the 
references therin; we will call them shrinking. We briefly recall some results and properties of maps in this class:

(1) the fixed point, if it exists, is unique;

(2) if $M$ is a compact set (or more generally if $\overline{T M}$ is compact), then $T$ has a fixed point $x^{*}$, and moreover for each $x \in M, T^{n} x \rightarrow x^{*}$;

(3) there is an example (see [5]) of a map on the unit ball of Hilbert spaces with fixed point $x^{*}$ such that $T^{n} x$ does not converge to the fixed point for any $x \neq x^{*}$;

(4) there are examples of maps without fixed points $[4,6,9]$.

Not so much attention has been paid to shrinking maps; indeed the following questions are open. Let $M$ be a weakly compact convex of a Banach space and let $T: M \rightarrow M$ be a shrinking mapping. Must $T$ have a fixed point? If $T$ has a fixed point $x^{*}$, is it true that $T^{n} x \rightarrow x^{*}$ for every $x$ ?

Conditions stronger than (S) were considered, also in more general settings, see for example [3]. Another rather weak strengthening, which appeared probably for the first time in [2], is the one given by the following definition. $T$ is diametrically contractive (DC) if $\delta(T(A))<\delta(A)$ for every closed, convex, bounded nonsingleton subset $A$ of $M$, where $\delta(A)$ is the diameter of $A$.

Such a notion was studied in details in [10]. We collect some relations between the previous classes of mappings:

(1) diametrically contractive maps are shrinking;

(2) if $M$ is a compact set and $T$ is shrinking, then it is diametrically contractive;

(3) there are examples of shrinking maps that are not diametrically contractive $[4,10]$.

A most important result is the following, see [10, Theorem 2.3].

Theorem 1.1. Let $M$ be a weakly compact subset of a Banach space $X$ and let $T: M \rightarrow M$ be diametrically contractive, then $T$ has a fixed point.

The proof of this theorem appeared probably for the first time in [7, Theorem 2] and in the case of reflexive spaces can be found in $[1,8]$.

The following problems appear to be open (see [10]).

Problem 1.2. Can we substitute weakly compact subset with closed convex bounded one in Theorem 1.1?

Problem 1.3. If $T$ is diametrically contractive and $x^{*}$ is the fixed point of $T$, do we have $T^{n} x \rightarrow x^{*}$ for all (or at least for some) $x \in M$ ?

In this paper, we solve in the negative both problems: the first example (Section 2) solves Problem 1.2; the second example (Section 3) solves Problem 1.3.

\section{First example}

Now we give an example of a fixed point free DC self-map of a closed convex bounded set. 
Consider the vector space of all continuous real functions on the closed unit interval, with the norm (equivalent to the classical one)

$$
\|f\|=\|f\|_{\infty}+\|f\|_{1}=\max _{0 \leq x \leq 1}|f(x)|+\int_{0}^{1}|f(x)| d x .
$$

Let $M=\{f \in X: f(0)=0 ; f(1)=1 ; 0 \leq f(x) \leq x ; f$ is monotone nondecreasing $\}$.

Define $T: M \rightarrow M$ in the following way:

$$
T f(x)= \begin{cases}0 & 0 \leq x \leq \frac{1}{2} \\ (2 x-1) f(2 x-1) & \frac{1}{2} \leq x \leq 1 .\end{cases}
$$

Claim 2.1. The map $T$ is fixed point free.

Proof. Suppose that $f \in M$ is such that $T f=f$. Clearly $f(x)=0$ for every $x \in[0 ; 1 / 2]$. If $x \in[1 / 2 ; 1]$, then $(2 x-1) f(2 x-1)=f(x)$ implies that $f(x)=0$ for every $x \in[0 ; 3 / 4]$. By iterating the reasoning, we can easily prove that $f(x)=0$ for all $x \in\left[0 ; 1-1 / 2^{n}\right]$ and all $n \in \mathbb{N}$. Since $f$ is continuous and $f(1)=1$, this is a contradiction proving the claim.

Claim 2.2. The map $T$ is shrinking.

Proof. Let be $f, g \in M$ with $f \neq g$. Then

$$
\begin{aligned}
\|T f-T g\|= & \max _{0 \leq x \leq 1}|T f(x)-T g(x)|+\int_{0}^{1}|T f(x)-T g(x)| d x \\
= & \max _{1 / 2 \leq x \leq 1}(2 x-1)|(f(2 x-1)-g(2 x-1))| \\
& +\int_{1 / 2}^{1}(2 x-1)|f(2 x-1)-g(2 x-1)| d x \\
= & \max _{0 \leq x \leq 1}|x(f(x)-g(x))|+\frac{1}{2} \int_{0}^{1} x|f(x)-g(x)| d x \\
< & \|f-g\|_{\infty}+\frac{1}{2}\|f-g\|_{1} \leq\|f-g\| .
\end{aligned}
$$

Claim 2.3. The map $T$ is diametrically contractive.

Proof. Let $A$ be a closed subset of $M$ such that $\delta(A)>0$. We have, for two suitable subsequences $f_{n}, g_{n}$,

$$
\begin{aligned}
\delta(T(A)) & =\lim _{n \rightarrow \infty}\left\|T f_{n}-T g_{n}\right\|=\lim _{n \rightarrow \infty}\left(\left\|T f_{n}-T g_{n}\right\|_{\infty}+\left\|T f_{n}-T g_{n}\right\|_{1}\right) \\
& \leq \lim _{n \rightarrow \infty}\left(\left\|f_{n}-g_{n}\right\|_{\infty}+\frac{1}{2}\left\|f_{n}-g_{n}\right\|_{1}\right) \leq \lim _{n \rightarrow \infty}\left\|f_{n}-g_{n}\right\| \leq \delta(A) .
\end{aligned}
$$

So, if we assume that $\delta(T(A))=\delta(A)$, then (by passing again if necessary to a subsequence) we have

$$
\begin{gathered}
\lim _{n \rightarrow \infty}\left\|f_{n}-g_{n}\right\|_{1}=\lim _{n \rightarrow \infty}\left\|T f_{n}-T g_{n}\right\|_{1}=0, \\
\lim _{n \rightarrow \infty}\left\|f_{n}-g_{n}\right\|_{\infty}=\lim _{n \rightarrow \infty}\left\|T f_{n}-T g_{n}\right\|_{\infty}=\delta(T(A))=\delta(A) .
\end{gathered}
$$


4 Diametrically contractive maps and fixed points

But we can choose a sequence $\left(x_{n}\right)$ such that $\left\|T f_{n}-T g_{n}\right\|_{\infty}=x_{n}\left|f_{n}\left(x_{n}\right)-g_{n}\left(x_{n}\right)\right|$. By considering eventually a subsequence, we may assume that $x_{n} \rightarrow x_{o} \in[0 ; 1]$. Then

$$
\delta(A)=\lim _{n \rightarrow \infty} x_{n}\left|f_{n}\left(x_{n}\right)-g_{n}\left(x_{n}\right)\right| \leq \lim _{n \rightarrow \infty} x_{n}|| f_{n}-g_{n} \|_{\infty}=x_{o} \delta(A),
$$

thus $x_{o}=1$.

By considering subsequences, and by exchanging eventually the sequences, we may assume that

$$
f_{n}\left(x_{n}\right) \longrightarrow l, \quad g_{n}\left(x_{n}\right) \longrightarrow L
$$

with $L \leq l \leq 1$.

Therefore (2.6) implies that

$$
l-L=\delta(A)
$$

so

$$
f_{n}\left(x_{n}\right) \longrightarrow l, \quad g_{n}\left(x_{n}\right) \longrightarrow l-\delta(A) .
$$

Now take any $f \in A$; since $\lim _{n \rightarrow \infty} x_{n}=1$, we have

$$
\delta(A) \geq\left|f\left(x_{n}\right)-g_{n}\left(x_{n}\right)\right| \underset{n \rightarrow \infty}{\longrightarrow}|1-l+\delta(A)| \geq \delta(A) .
$$

Thus we have $l=1 ; \lim _{n \rightarrow \infty}\left|f\left(x_{n}\right)-g_{n}\left(x_{n}\right)\right|=\delta(A)$ for every $f \in A$, and then

$$
\lim _{n \rightarrow \infty}\left\|f-g_{n}\right\|_{\infty}=\delta(A) .
$$

Now take $\epsilon \in(0, \delta(A))$, then there exists $\eta>0$ such that for every $x \in[1-\eta, 1]$, we have $1-\epsilon \leq f(x) \leq 1$. For $n$ large, $x_{n}>1-\eta$; therefore, by using also the monotonicity assumption for the functions, we have (for suitable points $c_{n}$ )

$$
\begin{aligned}
\int_{0}^{1}\left|f(x)-g_{n}(x)\right| d x & \geq \int_{1-\eta}^{x_{n}}\left|f(x)-g_{n}(x)\right| d x=\left(x_{n}-1+\eta\right)\left|f\left(c_{n}\right)-g_{n}\left(c_{n}\right)\right| \\
& \geq\left(x_{n}-1+\eta\right)\left(1-\epsilon-g_{n}\left(x_{n}\right)\right) ;
\end{aligned}
$$

also, since $\lim _{n \rightarrow \infty} g_{n}\left(x_{n}\right)=1-\delta(A)$,

$$
\lim _{n \rightarrow \infty}\left(x_{n}-1+\eta\right)\left(1-\epsilon-g_{n}\left(x_{n}\right)\right)=\eta(\delta(A)-\epsilon) .
$$

Thus we obtain

$$
\liminf _{n \rightarrow \infty}\left\|f-g_{n}\right\|_{1} \geq \eta(\delta(A)-\epsilon)
$$

and this implies that

$$
\liminf _{n \rightarrow \infty}\left\|f-g_{n}\right\| \geq \lim _{n \rightarrow \infty}\left\|f-g_{n}\right\|_{\infty}+\liminf _{n \rightarrow \infty}\left\|f-g_{n}\right\|_{1} \geq \delta(A)+\eta(\delta(A)-\epsilon) .
$$

This is a contradiction, proving the claim and thus the result. 


\section{Second example}

The next example shows that for a DC self-map of a bounded closed convex set $M$, the existence of a fixed point does not imply the convergence of iterates $T^{n} x$ to the fixed point.

Consider the vector space $c_{o}$, endowed with the following norm (equivalent to the usual one):

$$
\|x\|=\|x\|_{\infty}+\sum_{n=1}^{\infty} \frac{\left|x_{n}\right|}{2^{n}} .
$$

We denote by $B^{+}$the intersection of the positive cone with the unit closed ball. Define $T: B^{+} \rightarrow B^{+}$in this way:

$$
(T x)_{1}=0, \quad \text { for } n \geq 2, \quad(T x)_{n}=a_{n-1} x_{n-1},
$$

where $\left(a_{n}\right), n \geq 1$, is a strictly positive and strictly increasing sequence such that $\prod_{n=1}^{\infty} a_{n}=$ $\alpha>0$. Clearly $T$ is linear and its unique fixed point is the null vector.

The map $T$ is shrinking: in fact, for $x \neq y$,

$$
\begin{aligned}
\|T x-T y\| & =\left\|\left(0, a_{1}\left(x_{1}-y_{1}\right), a_{2}\left(x_{2}-y_{2}\right), \ldots\right)\right\| \\
& <\left\|\left(0,\left(x_{1}-y_{1}\right),\left(x_{2}-y_{2}\right), \ldots\right)\right\|<\|x-y\| .
\end{aligned}
$$

Consider now the orbit of non-null elements in $B^{+}$. Take $x$ and let for example $x_{k} \neq 0$. We have

$$
\left\|T^{n} x\right\| \geq\left|\left(T^{n} x\right)_{k+n}\right|=a_{k} a_{k+1} \cdots a_{k+n-1} x_{k} \underset{n \rightarrow \infty}{\longrightarrow}\left(\prod_{n=k}^{\infty} a_{n}\right) \quad x_{k} \neq 0 .
$$

Now we will prove that our map $T$ is diametrically contractive.

Consider a bounded closed convex set $A$ contained in $B^{+}$. Let us suppose that

$$
\delta(A)=\delta(T(A))>0 .
$$

Consider two sequences $x^{(n)}$ and $y^{(n)}$ such that

$$
\lim _{n \rightarrow \infty}\left\|T x^{(n)}-T y^{(n)}\right\|=\delta(T(A))
$$


6 Diametrically contractive maps and fixed points

Since $T$ is shrinking, this implies that $\lim _{n \rightarrow \infty}\left\|x^{(n)}-y^{(n)}\right\|=\delta(A)$. We have

$$
\begin{aligned}
\delta(T(A)) & =\lim _{n \rightarrow \infty}\left(\left\|T\left(x^{(n)}-y^{(n)}\right)\right\|_{\infty}+\sum_{k=1}^{\infty} \frac{\left|T\left(\left(x^{(n)}\right)-\left(y^{(n)}\right)\right)_{k}\right|}{2^{k}}\right) \\
& =\lim _{n \rightarrow \infty}\left(\max _{k \geq 2}\left|a_{k-1}\left(x_{k-1}^{(n)}-y_{k-1}^{(n)}\right)\right|+\sum_{k=2}^{\infty} \frac{a_{k-1}\left|x_{k-1}^{(n)}-y_{k-1}^{(n)}\right|}{2^{k}}\right) \\
& =\lim _{n \rightarrow \infty}\left(\max _{k \geq 1} a_{k}\left|x_{k}^{(n)}-y_{k}^{(n)}\right|+\sum_{k=1}^{\infty} \frac{a_{k}\left|x_{k}^{(n)}-y_{k}^{(n)}\right|}{2^{k+1}}\right) \\
& \leq \limsup _{n \rightarrow \infty}\left(\left\|x^{(n)}-y^{(n)}\right\|_{\infty}+\frac{1}{2} \sum_{k=1}^{\infty} \frac{\left|x_{k}^{(n)}-y_{k}^{(n)}\right|}{2^{k}}\right) \leq \delta(A) .
\end{aligned}
$$

From this, we obtain

$$
\begin{gathered}
\lim _{n \rightarrow \infty}|| x^{(n)}-y^{(n)} \|_{\infty}=\delta(A), \\
\lim _{n \rightarrow \infty} \sum_{k=1}^{\infty} \frac{\left|x_{k}^{(n)}-y_{k}^{(n)}\right|}{2^{k}}=0 .
\end{gathered}
$$

For every $n$, there exists $k(n)$ such that $\left\|x^{(n)}-y^{(n)}\right\|_{\infty}=\left|x_{k(n)}^{(n)}-y_{k(n)}^{(n)}\right|$, so

$$
\lim _{n \rightarrow \infty}\left|x_{k(n)}^{(n)}-y_{k(n)}^{(n)}\right|=\delta(A)
$$

Set $K=\{k(n) ; n \in \mathbb{N}\}$. If $K$ is finite, then $k(n)=k_{o}$ for infinitely many $n$, so

$$
\sum_{k=1}^{\infty} \frac{\left|x_{k}^{(n)}-y_{k}^{(n)}\right|}{2^{k}} \geq \frac{\left|x_{k_{o}}^{(n)}-y_{k_{o}}^{(n)}\right|}{2^{k_{o}}} \underset{n \rightarrow \infty}{\longrightarrow} \frac{\delta(A)}{2^{k_{o}}} \neq 0
$$

which is an absurdity since we have proved that the left-hand side tends to 0 . Thus $K$ is infinite. Take a subsequence of $k(n)$ tending to infinity, that we still call $k(n)$, such that $x_{k(n)}^{(n)} \rightarrow \delta(A)+l$ and $y_{k(n)}^{(n)} \rightarrow l(\geq 0)$.

Now let $x \in A$; we have

$$
\delta(A)+l=\lim _{n \rightarrow \infty}\left|x_{k(n)}-x_{k(n)}^{(n)}\right| \leq \lim _{n \rightarrow \infty}\left\|x-x^{(n)}\right\|_{\infty} \leq \lim _{n \rightarrow \infty}\left\|x-x^{(n)}\right\| \leq \delta(A)
$$

This implies that $l=0$.

Therefore, for every $x \in A, \lim _{n \rightarrow \infty}\left\|x-x^{(n)}\right\|=\delta(A)$. So

$$
\lim _{n \rightarrow \infty} \sum_{k=1}^{\infty} \frac{\left|x_{k}-x_{k}^{(n)}\right|}{2^{k}}=0
$$


which implies, for every $k$, that

$$
\lim _{n \rightarrow \infty} x_{k}^{(n)}=x_{k},
$$

(remember that this should be true for every $x \in A$ ) so $A$ cannot contain two or more elements. This would imply $\delta(A)=0$, against the assumption. This contradiction proves the assertion.

\section{Final remarks}

After discussing Problems 1.2 and 1.3, another rather awkward condition, stronger than DC, was introduced in [10].

Given a set $M$, say that $T: M \rightarrow M$ is asymptotically diametrically contractive ADC if for all nested sequences $\left(A_{n}\right)$ of closed bounded subsets of $M$ with $\lim _{n \rightarrow \infty} \delta\left(A_{n}\right)=\delta>0$, we have $\lim _{n \rightarrow \infty} \delta\left(T\left(A_{n}\right)\right)<\delta$.

We try to clarify its position among other simpler conditions.

Clearly, ADC maps are DC; as proved in [10, Theorem 2.6], the following result holds. If $T: M \rightarrow M$ is an ADC map and $T$ has a bounded orbit for some $x_{o} \in M$, then $T$ has a unique fixed point $\xi$, and for every $x \in M: T^{n}(x) \rightarrow \xi$. In particular, this fact is true whenever $M$ is bounded.

If $M$ is compact, then (S) implies DC and DC implies ADC. But there are (S) maps on compact sets which are not contractive; thus ADC does not imply contractiveness, also when the map is defined on a compact set. An example of a map, on an unbounded set, which is ADC but not contractive, was given in [10, Remark 2.7].

An example of a map satisfying (S), but which is not DC, was given in [10]; according to the previous result, our first and second examples (Sections 2 and 3) show that DC maps are not in general ADC.

\section{References}

[1] L. Ćirić, A fixed-point theorem in reflexive Banach spaces, Publications de Institut Mathématique (Beograd). Nouvelle Série 36(50) (1984), 105-106.

[2] V. I. Istrateşcu, Some fixed theorems for convex contraction mappings and mappings with convex diminishing diameters. IV Nonexpansive diameter mappings in uniformly convex spaces, Preliminary report. Abstracts of the American Mathematical Society (1982), 82T-46-316.

[3] J. Jachymski, Order-theoretic aspects of metric fixed point theory, Handbook of Metric Fixed Point Theory (W. A. Kirk and B. Sims, eds.), Kluwer Academic, Dordrecht, 2001, pp. 613-641.

[4] W. A. Kirk, A fixed point theorem for mappings which do not increase distances, The American Mathematical Monthly 72 (1965), no. 9, 1004-1006.

[5] S. B. Nadler Jr., A note on an iterative test of Edelstein, Canadian Mathematical Bulletin 15 (1972), 381-386.

[6] I. Rosenholtz, On a fixed point problem of D. R. Smart, Proceedings of the American Mathematical Society 55 (1976), no. 1, 252.

[7] K. P. R. Sastry and S. V. R. Naidu, Some fixed point theorems in normed linear spaces, Indian Journal of Pure and Applied Mathematics 10 (1979), no. 8, 928-937.

[8] V. M. Sehgal and S. P. Singh, A fixed point theorem in reflexive Banach spaces, Mathematics Seminar Notes. Kobe University 11 (1983), no. 1, 81-82. 
[9] B. Sims, Examples of fixed point free mappings, Handbook of Metric Fixed Point Theory (W. A. Kirk and B. Sims, eds.), Kluwer Academic, Dordrecht, 2001, pp. 35-48.

[10] H.-K. Xu, Diametrically contractive mappings, Bulletin of the Australian Mathematical Society 70 (2004), no. 3, 463-468.

Marco Baronti: Sezione Metodi e Modelli Matematici Dipartimento di Ingegneria della Produzione, Termoenergetica e Metodi e Modelli Matematici, Università degli Studi di Genova, Piazzale Kennedy, Genova 16129, Italy

E-mail address: baronti@dimet.unige.it

Emanuele Casini: Dipartimento di Fisica e Matematica, Università dell'Insubria, Via Valleggio 11, Como 22100, Italy

E-mail address: emanuele.casini@uninsubria.it

Pier Luigi Papini: Dipartimento di Matematica, Università degli Studi di Bologna,

Piazza Porta S. Donato 5, Bologna 40126, Italy

E-mail address: papini@dm.unibo.it 